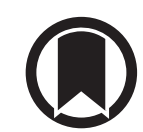

CrossMark

\title{
Effects of short-term continuous positive airway pressure withdrawal on cerebral vascular reactivity measured by blood oxygen level-dependent magnetic resonance imaging in obstructive sleep apnoea: a randomised controlled trial
}

\author{
Sira Thiel ${ }^{1,8}$, Franziska Lettau $^{1,8}$, Protazy Rejmer $^{1}$, Cristina Rossi $^{2}$, \\ Sarah R. Haile ${ }^{3}$, Esther I. Schwarz ${ }^{1}$, Anna S. Stöberl ${ }^{1}$, Noriane A. Sievi ${ }^{1}$, \\ Andreas Boss ${ }^{4}$, Anton S. Becker $\mathbb{1}^{4}$, Sebastian Winklhofer ${ }^{5}$, John R. Stradling ${ }^{6}$ \\ and Malcolm Kohler ${ }^{1,7}$
}

@ERSpublications

Withdrawing continuous positive airway pressure therapy and the resulting recurrence of obstructive sleep apnoea did not result in a significant reduction in cerebral vascular reactivity, despite clinically relevant increases in blood pressure http://ow.ly/T2QD30mU6L2

Cite this article as: Thiel S, Lettau F, Rejmer P, et al. Effects of short-term continuous positive airway pressure withdrawal on cerebral vascular reactivity measured by blood oxygen level-dependent magnetic resonance imaging in obstructive sleep apnoea: a randomised controlled trial. Eur Respir J 2019; 53: 1801854 [https://doi.org/10.1183/13993003.01854-2018].

ABSTRACT Impaired cerebral vascular reactivity (CVR) increases long-term stroke risk. Obstructive sleep apnoea (OSA) is associated with peripheral vascular dysfunction and vascular events. The aim of this trial was to evaluate the effect of continuous positive airway pressure (CPAP) withdrawal on CVR.

41 OSA patients ( $88 \%$ male, mean age $57 \pm 10$ years) were randomised to either subtherapeutic or continuation of therapeutic CPAP. At baseline and after 2 weeks, patients underwent a sleep study and magnetic resonance imaging (MRI). CVR was estimated by quantifying the blood oxygen level-dependent (BOLD) MRI response to breathing stimuli.

OSA did recur in the subtherapeutic CPAP group (mean treatment effect apnoea-hypopnoea index +38.0 events $\mathrm{h}^{-1}, 95 \%$ CI 24.2-52.0; $\mathrm{p}<0.001$ ) but remained controlled in the therapeutic group. Although there was a significant increase in blood pressure upon CPAP withdrawal (mean treatment effect $+9.37 \mathrm{mmHg}, 95 \%$ CI 1.36-17.39; $\mathrm{p}=0.023$ ), there was no significant effect of CPAP withdrawal on CVR assessed via BOLD MRI under either hyperoxic or hypercapnic conditions.

Short-term CPAP withdrawal did not result in statistically significant changes in CVR as assessed by functional MRI, despite the recurrence of OSA. We thus conclude that, unlike peripheral endothelial function, CVR is not affected by short-term CPAP withdrawal.

This article has supplementary material available from erj.ersjournals.com

This study is registered at clinicaltrials.gov with identifier NCT02493673. Data are available from the Dept of Pulmonology, University Hospital Zurich, after approval from the Local Ethics Committee Zurich, Switzerland, and from the applicants' own institution, and with agreement from the PI of the study, M. Kohler. Due to ethical reasons and the privacy of the patients, the data are available upon request. Requests for the data may be sent to the corresponding author.

Received: July 192018 | Accepted after revision: Nov 202018

Copyright @ERS 2019 


\section{Introduction}

In population-based studies, the prevalence of moderate-to-severe sleep-disordered breathing in the middle-aged population is estimated to be between $9 \%$ and $23 \%$ in women and between $17 \%$ and $50 \%$ in men $[1,2]$. Obstructive sleep apnoea (OSA), characterised by complete or partial obstruction of the upper airway, causes intermittent hypoxia, hypercapnia, increased sympathetic nervous system activity, surges in blood pressure (BP) and impaired peripheral vascular function [3, 4]. OSA is associated with increased risk of stroke, cardiovascular events, heart failure and impaired neurocognitive function [5-7]. However, the underlying pathophysiological mechanisms are poorly understood. Contrasting findings exist about cerebral vascular reactivity (CVR) and cerebral blood flow (CBF) deregulation in OSA patients. How these impairments contribute to the risk of stroke remains to be clarified. The use of different imaging techniques and statistical thresholds and the lack of standardised measurement methods make it difficult to compare results of previous studies [8-17].

Functional magnetic resonance imaging (MRI) during respiratory challenges allows estimation of CVR and CBF [18]. Blood oxygenation level-dependent (BOLD) MRI is able to detect magnetic field variations induced by changes in oxyhaemoglobin and deoxyhaemoglobin concentrations. The BOLD signal responds to changes in the arterial gas concentration induced by the administration of gas mixtures or breath holding [18]. While pure oxygen inhalation mostly induces a change in the deoxyhaemoglobin concentration and, in turn, a BOLD signal increase, the administration of gas mixtures containing carbon dioxide $\left(\mathrm{CO}_{2}\right)$ results in an additional vascular modulation of the BOLD response [19].

Continuous positive airway pressure (CPAP) therapy is sometimes interrupted, e.g. during upper airway infections or during holidays. These interruptions, and thus recurrence of OSA, might impair CVR and CBF by several possible mechanisms, e.g. impaired endothelial function, augmented sympathetic activity and an increase in oxidative stress due to intermittent hypoxia as well as reduced cerebral tissue oxygenation $[3,20]$. During apnoeic episodes, increases in intracranial pressure, correlating with systemic BP fluctuations, could result in an excess of flow in brain vessels following apnoea termination, leading to capillary damage, because brain tissue is sensitive to rapid reperfusion [21]. Information on CVR in OSA patients assessed via BOLD MRI is sparse and originates mainly from small and mostly case-control studies $[8,11,15,17]$.

We conducted a 2-week CPAP withdrawal randomised controlled trial in patients with moderate-to-severe OSA to examine the link between OSA, CVR and brain perfusion, respectively. We hypothesised that CPAP withdrawal would result in a reduction of daytime CVR and CBF.

\section{Methods}

\section{Trial design}

This randomised, double-blind, placebo-controlled, parallel-group trial (therapeutic versus subtherapeutic CPAP) included 41 patients with moderate-to-severe OSA. Patients had been treated with CPAP for at least 1 year. Dynamic MRI acquisitions were performed between 07:00 $\mathrm{h}$ and 09:00 $\mathrm{h}$ during the inhalation of medical air (MA) (i.e. $\left.21 \% \mathrm{O}_{2}\right)$, oxygen $\left(99.5 \% \mathrm{O}_{2}\right)$ and carbogen $\left(5 \% \mathrm{CO}_{2}+95 \% \mathrm{O}_{2}\right)$. We refer to the conditions induced by MA, oxygen and carbogen as "normoxia", "hyperoxia" and "hypercapnia", respectively. Gases were administered in blocks of 3 min each (MA-oxygen-MA-oxygen-MA-carbogen-MA). Participants wore a mask with a one-way valve and a 0.5 - $\mathrm{L}$ reservoir bag; the mask covered the mouth and nose completely and was tightly adjusted to the face. We requested that the subjects breathed normally; gas flow rates were set to $10 \mathrm{~L} \cdot \mathrm{min}^{-1}$ each (supplementary figure e1).

\section{Subjects}

Participants were eligible if they met the following inclusion criteria: 1) age between 20 and 75 years; 2) apnoea-hypopnoea index (AHI) and/or oxygen desaturation index (ODI $4 \%) \geqslant 20$ events. $\mathrm{h}^{-1}$ in their in-laboratory sleep study at the time of diagnosis; 3) treated with CPAP for at least 1 year with high

Affiliations: ${ }^{1}$ Dept of Pulmonology and Sleep Disorders Centre, University Hospital Zurich, Zurich, Switzerland. ${ }^{2}$ Dept of Diagnostic and Interventional Radiology, University Hospital Zurich, Zurich, Switzerland. ${ }^{3}$ Dept of Epidemiology, Biostatistics and Prevention Institute, University of Zurich, Zurich, Switzerland. ${ }^{4}$ Dept of Diagnostic and Interventional Radiology, University Hospital Zurich, Zurich, Switzerland. ${ }^{5}$ Dept of Neuroradiology, University Hospital Zurich, Zurich, Switzerland. ${ }^{6}$ National Institute for Health Research (NIHR) Oxford Biomedical Research Centre, Oxford University Hospitals NHS Foundation Trust and University of Oxford, Oxford, UK. ${ }^{7}$ Centre for Integrative Human Physiology, University of Zurich, Zurich, Switzerland. ${ }^{8}$ Contributed equally.

Correspondence: Malcolm Kohler, Division of Pulmonology and Sleep Disorders Centre, University Hospital of Zurich, Raemistrasse 100, Zurich, Switzerland. E-mail: malcolm.kohler@usz.ch 
compliance (device usage $\geqslant 4 \mathrm{~h}$ per night on at least $80 \%$ of the past 365 days with a current AHI $\leqslant 10$ events $\mathrm{h}^{-1}$ on treatment, measured from the CPAP machine download data); and 4) an ODI $4 \% \geqslant 15$ events $\mathrm{h}^{-1}$ from current nocturnal pulse oximetry studies during a preliminary 5 -night period off CPAP treatment. The trial was approved by the local ethics committee (KEK-ZH-No.2014-0684), and all procedures in this trial involving human participants were performed in accordance with Good Clinical Practice guidelines. The trial was registered prior to commencement (ClinicalTrials.gov identifier: NCT02493673). See supplementary material for details.

\section{Patient evaluation and follow-up}

Recruitment started in June 2015 and the last follow-up was completed in December 2017. Once the persistence of relevant OSA was confirmed (number of oxygen desaturations $\geqslant 4 \%$ (ODI4\%) $\geqslant 15$ events. $\mathrm{h}^{-1}$ ) by home overnight pulse oximetry (Pulsox-300i; Konica Minolta Sensing Inc., Osaka, Japan) during the preliminary 5-night period off CPAP, patients resumed CPAP therapy for at least 2 weeks. An MS-DOS program (MINIM, London, UK) allocated participants using two minimisation criteria: ODI $4 \%$ $<30$ or $>30$ events $\cdot \mathrm{h}^{-1}$ and body mass index $<35$ or $>35 \mathrm{~kg} \cdot \mathrm{m}^{-2}$. Baseline in-laboratory assessments were performed on all subjects using therapeutic CPAP. Follow-up assessments were performed after 2 weeks on either therapeutic (control arm) or subtherapeutic (intervention arm) CPAP settings. Participants as well as outcome assessors remained blinded to the treatment assignment until completion of the data analysis.

\section{Sleep studies and CPAP devices}

In-hospital respiratory polygraphies (Alice 5 Diagnostics System; Philips Respironics, Pittsburgh, PA, USA) were scored manually according to the American Academy of Sleep Medicine task force criteria [22]. The severity of OSA was quantified using the AHI and the ODI4\%. Patients in both groups received the same CPAP device (REMstar Auto A-Flex; Philips Respironics). In the therapeutic group, pressure and mode were set according to the previous individual's settings. In the withdrawal group, subtherapeutic pressure was generated by setting the CPAP device to the lowest pressure, inserting a flow-restricting connector at the machine outlet, and inserting six extra holes in the collar of the tube at the end of the mask to prevent rebreathing of $\mathrm{CO}_{2}$ (supplementary figures e1 and e2).

\section{Primary outcome}

The primary outcome was CVR in response to hyperoxia and hypercapnia of grey matter (GM), white matter (WM) and the whole brain assessed by functional MRI as measures of cerebral endothelial function. See supplementary material for details.

\section{Secondary outcome measures}

Before gas administration, CBF was assessed using arterial spin labelling (ASL) MRI.

Participants measured their $\mathrm{BP}$ and heart rate $(\mathrm{HR})$ in triplicate every morning; the average of three measurements was used for further analysis.

Subjective sleepiness was assessed using the Epworth Sleepiness Score. See supplementary material for details.

\section{Statistical methods/data analysis}

Normally distributed data are expressed as mean \pm sD, unless stated otherwise. For all outcomes, we calculated an effect size and 95\% confidence intervals with a linear regression analysis adjusting for treatment group and baseline measurements of the outcome. A multivariable linear regression adjusting for several variables (e.g. sex, age, BP at home and in hospital, AHI, ODI) was also performed to generate and adjust effect size; a two-sided significance level of $<0.05$ was used to determine statistical significance. The statistical analysis was performed in $\mathrm{R}$ (version 3.4.4; $\mathrm{R}$ Core Team, Vienna, Austria). See the supplementary material for more information (supplementary table e1).

\section{Results}

Trial profile and patient characteristics

The trial flow chart is presented in figure 1. 49 patients were randomised and allocated to therapeutic $(n=27)$ or subtherapeutic $(n=22)$ CPAP for 2 weeks. The two trial arms were similar regarding baseline patient characteristics (table 1).

\section{Effects of CPAP withdrawal on cerebral vascular reactivity}

There was no significant effect of short-term CPAP withdrawal on CVR assessed via BOLD MRI in GM, WM or whole brain under either hyperoxic or hypercapnic conditions (table 2, supplementary table e2, 
supplementary figures e3-e5). As expected, the quantitative BOLD response analysis showed an effect on the signal pattern depending on the applied stimulus and tissue type (figures 2 and 3 ).

Multivariable linear regression modelling to calculate the treatment effect size, adjusting for baseline measurements as well as age, sex, systolic BP, diastolic BP, HR, AHI and ODI, did not change the results significantly (supplementary tables e1 and e2).

\section{Effects of CPAP withdrawal on secondary outcomes}

Cerebral blood flow

A 2-week CPAP withdrawal was not associated with a significant change in CBF assessed via functional MRI over GM (mean treatment effect $+4.20 \mathrm{~mL} \cdot 100 \mathrm{~g} \cdot \mathrm{min}^{-1}, 95 \% \mathrm{CI}-0.96-9.36 ; \mathrm{p}=0.110$ ) or WM (mean treatment effect $-0.62 \mathrm{~mL} \cdot 100 \mathrm{~g} \cdot \mathrm{min}^{-1}, 95 \% \mathrm{CI}-3.90-2.66 ; \mathrm{p}=0.700$ ) (table 3 ).

\section{Subjective sleepiness}

CPAP withdrawal led to a statistically significant increase in the Epworth Sleepiness Score compared with continuing CPAP (mean treatment effect +3.29 , 95\% CI 0.87-5.72; $\mathrm{p}=0.009$ ) (table 3).

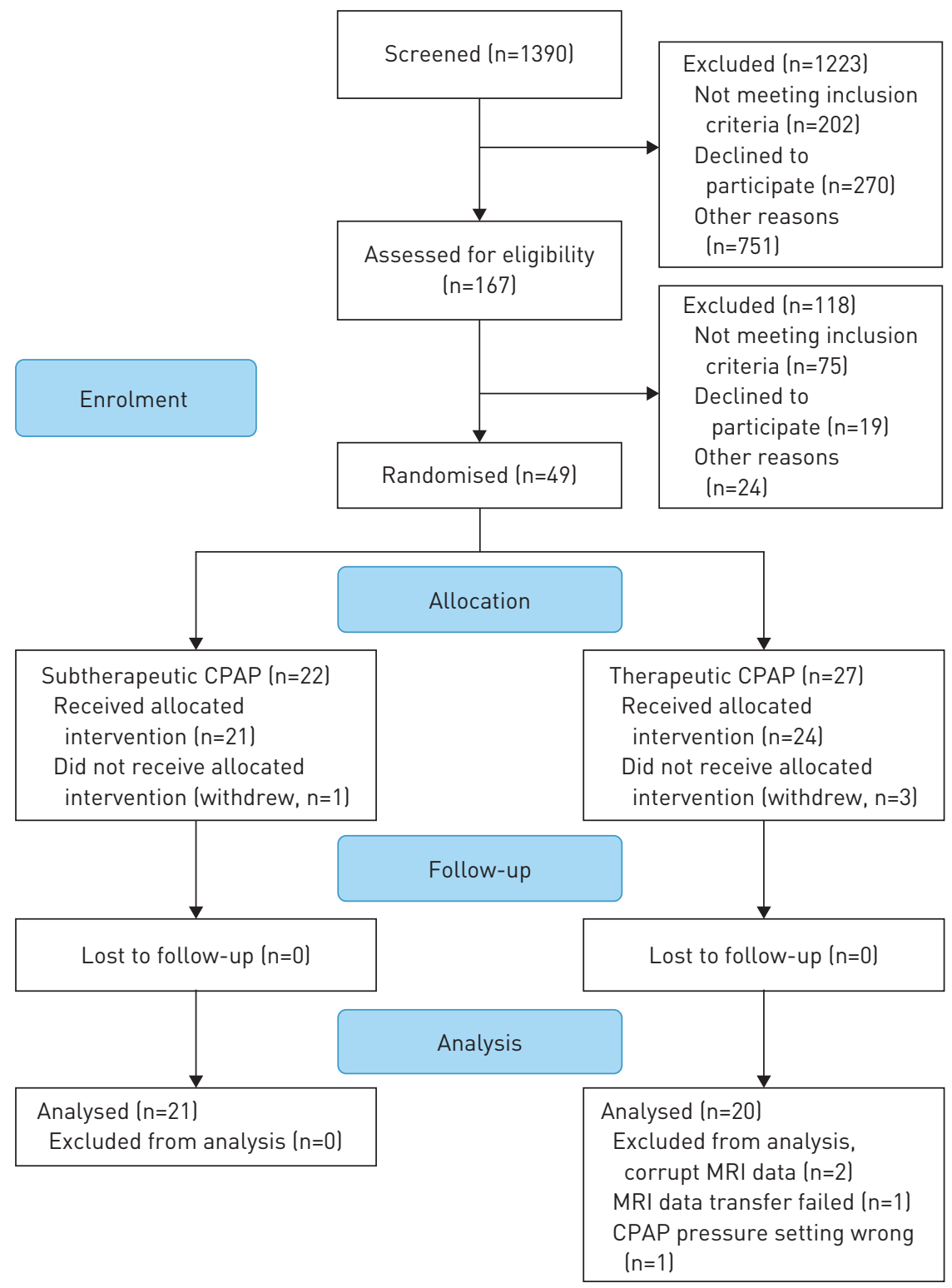

FIGURE 1 CONSORT flow diagram. CPAP: continuous positive airway pressure; MRI: magnetic resonance imaging. 
TABLE 1 Baseline characteristics of the trial population

\begin{tabular}{lcc} 
& Subtherapeutic CPAP & Therapeutic CPAP \\
\hline Subjects $\mathbf{n}$ & 21 & 20 \\
Age years & $56.2 \pm 9.2$ & $57.6 \pm 11.1$ \\
Male sex & $20(95)$ & $16(80)$ \\
BMI $\mathbf{k g} \cdot \mathbf{m}^{-2}$ & $35.1 \pm 5.6$ & $32.8 \pm 7.2$ \\
Comorbidities & & \\
Hypertension & $10(48)$ & $10(50)$ \\
Diabetes mellitus & $6(29)$ & $4(20)$ \\
Dyslipidaemia & $2(10)$ & $2(10)$ \\
Obesity & $17(81)$ & $14(70)$ \\
Medications & & \\
ACE inhibitor & $3(14)$ & $6(30)$ \\
AT 2 antagonist & $5(24)$ & $2(10)$ \\
Calcium channel blocker & $5(24)$ & $5(25)$ \\
Diuretics & $4(19)$ & $6(30)$ \\
Anit-diabetic drugs & $6(29)$ & $4(20)$ \\
Statins & $4(19)$ & $5(25)$ \\
Other & $10(48)$ & $15(75)$ \\
OSA severity and CPAP usage & & \\
ODI 5 nights off CPAP events $\cdot h^{-1}$ & $42.3 \pm 23.6$ & $37.0 \pm 16.2$ \\
AHI under CPAP events $\mathrm{h}^{-1}$ & $2.9 \pm 2.0$ & $2.1 \pm 2.2$ \\
CPAP usage \% days in 1 year & $94.8 \pm 6.9$ & $96.0 \pm 5.0$ \\
CPAP usage h & $6.1 \pm 1.3$ & $6.4 \pm 1.3$ \\
& &
\end{tabular}

Data are presented as $n(\%)$ or mean $\pm S D$, unless otherwise stated. CPAP: continuous positive airway pressure; BMI: body mass index; ACE: angiotensin converting enzyme; AT 2: angiotensin II blocker; OSA: obstructive sleep apnoea; AHI: apnoea-hypopnoea index; ODI: oxygen desaturation index.

Ambulatory blood pressure and heart rate

Discontinuation of CPAP for 2 weeks compared with continuing CPAP led to a statistically significant increase in systolic BP (mean treatment effect $+9.37 \mathrm{mmHg}, 95 \%$ CI 1.36-17.39; $\mathrm{p}=0.023$ ) and diastolic BP (mean treatment effect $+7.61 \mathrm{mmHg}, 95 \%$ CI $1.40-13.83 ; \mathrm{p}=0.018$ ). There was a trend towards an increase in HR in the subtherapeutic CPAP group (mean treatment effect $+4.01 \mathrm{bpm}, 95 \%$ CI $-0.37-8.40 ; \mathrm{p}=0.071$ ) (table 3).

Effects of CPAP withdrawal on obstructive sleep apnoea

Withdrawal of CPAP was associated with a return of OSA as demonstrated by a significant increase in AHI (mean treatment effect +38.0 events $\mathrm{h}^{-1}, 95 \%$ CI 24.2-52.0; $\mathrm{p}<0.0001$ ) and ODI (mean treatment effect +38.0 events $\cdot \mathrm{h}^{-1}, 95 \%$ CI 23.1-53.0; $\mathrm{p}<0.0001$ ) at 2 weeks (table 3 ).

TABLE 2 BOLD MRI signal changes during hyperoxic and hypercapnic challenges

\begin{tabular}{|c|c|c|c|c|c|c|c|c|c|}
\hline \multirow{2}{*}{$\begin{array}{c}\text { BOLD signal } \\
\text { change } \%\end{array}$} & & \multicolumn{3}{|c|}{ Subtherapeutic CPAP group\# } & \multicolumn{3}{|c|}{ Therapeutic CPAP group " } & \multirow[t]{2}{*}{ Treatment effect $^{+}$} & \multirow[t]{2}{*}{ p-value } \\
\hline & & Baseline & Follow-up & Change & Baseline & Follow-up & Change & & \\
\hline \multirow[t]{2}{*}{ Grey matter } & First $\mathrm{O}_{2}$ & $2.61 \pm 1.13$ & $2.57 \pm 0.87$ & $-0.04 \pm 1.14$ & $2.59 \pm 0.86$ & $3.11 \pm 1.01$ & $0.52 \pm 1.02$ & $-0.54(-1.10-0.02)$ & 0.056 \\
\hline & $\mathrm{CO}_{2}$ & $3.49 \pm 1.37$ & $3.47 \pm 1.21$ & $-0.02 \pm 1.18$ & $3.89 \pm 2.00$ & $3.52 \pm 1.12$ & $-0.37 \pm 2.00$ & $0.07(-0.61-0.75)$ & 0.84 \\
\hline \multirow[t]{2}{*}{ Overall brain } & First $\mathrm{O}_{2}$ & $2.36 \pm 0.81$ & $2.52 \pm 0.89$ & $0.16 \pm 0.66$ & $2.75 \pm 1.53$ & $2.72 \pm 0.50$ & $-0.03 \pm 1.56$ & $-0.12(-0.57-0.32)$ & 0.58 \\
\hline & Second $\mathrm{O}_{2}$ & $2.24 \pm 0.82$ & $2.30 \pm 0.79$ & $0.06 \pm 0.81$ & $2.47 \pm 1.03$ & $2.47 \pm 0.72$ & $0.00 \pm 1.11$ & $-0.11(-0.56-0.35)$ & 0.64 \\
\hline \multirow{2}{*}{ White matter } & Second $\mathrm{O}_{2}$ & $1.65 \pm 0.86$ & $1.68 \pm 0.97$ & $0.03 \pm 1.05$ & $1.41 \pm 0.84$ & $1.36 \pm 0.61$ & $-0.05 \pm 0.88$ & $0.24(-0.26-0.75)$ & 0.33 \\
\hline & $\mathrm{CO}_{2}$ & $1.90 \pm 0.71$ & $2.02 \pm 0.96$ & $0.12 \pm 1.09$ & $1.87 \pm 1.07$ & $1.81 \pm 0.92$ & $-0.07 \pm 1.36$ & $0.21(-0.39-0.81)$ & 0.48 \\
\hline
\end{tabular}

Data are presented as mean \pm SD or mean $(95 \% \mathrm{CI})$. BOLD: blood oxygen level-dependent; MRI: magnetic resonance imaging; CPAP: continuous positive airway pressure. ${ }^{\#}: \mathrm{n}=21 ;{ }^{\uparrow}: \mathrm{n}=20 ;{ }^{+}$: treatment effect (mean follow-up measurement in the subtherapeutic CPAP arm minus mean follow-up measurement in the therapeutic CPAP arm), adjusted for baseline of subtherapeutic CPAP. 

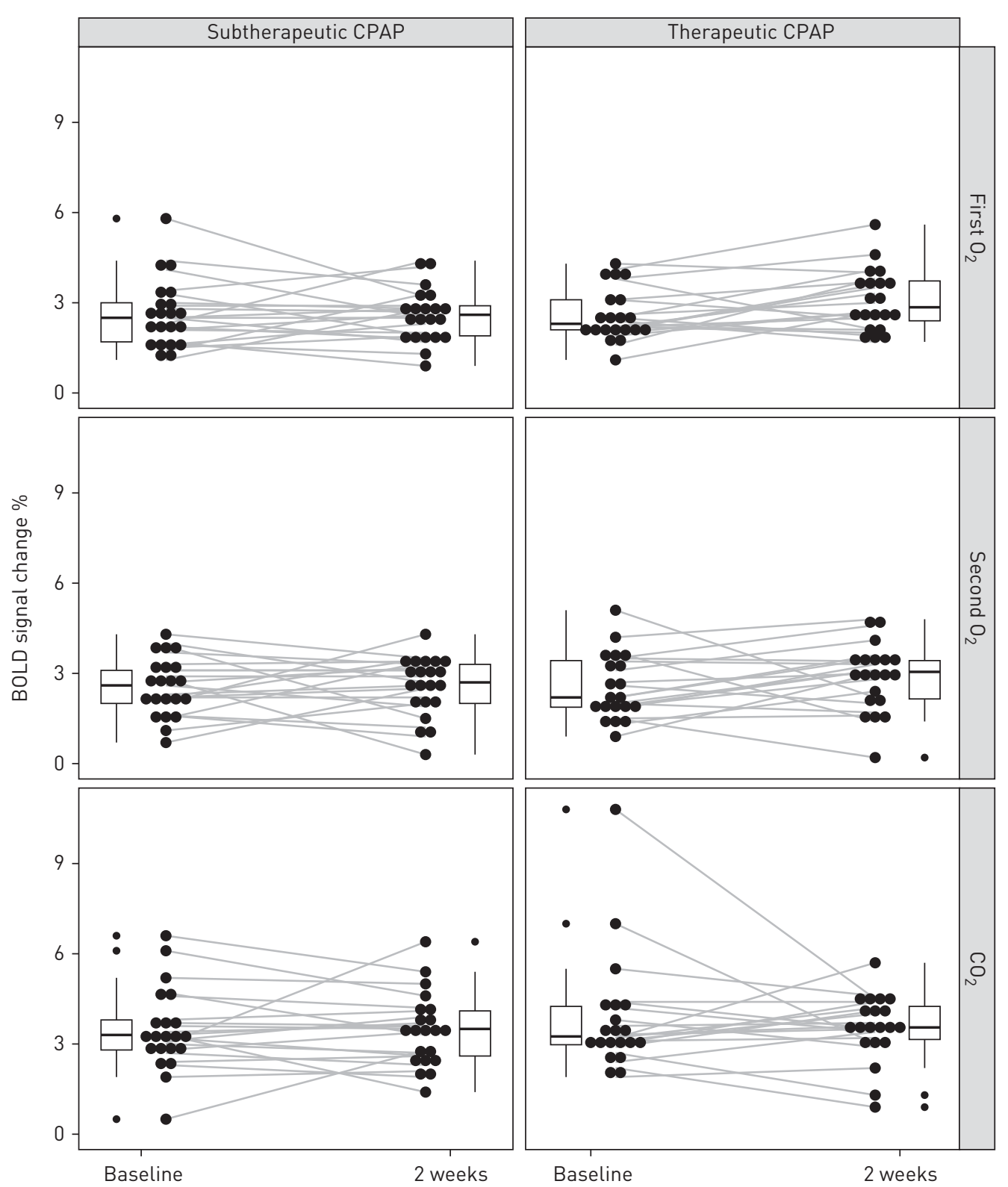

FIGURE 2 Percentage blood oxygen level-dependent (BOLD) signal change in grey matter. The subtherapeutic continuous positive airway pressure (CPAP) group is shown on the left and the therapeutic CPAP group on the right. Boxplots are shown for the first and second hyperoxic stimulus $\left(\mathrm{O}_{2}\right)$ as well as the hypercapnic stimulus $\left(\mathrm{CO}_{2}\right)$ at baseline and follow-up after 2 weeks.

\section{Discussion}

This randomised controlled trial investigated the possible changes in CVR and CBF induced by short-term withdrawal of CPAP in patients with moderate-to-severe OSA. The recurrence of OSA upon CPAP withdrawal was documented by a return of sleep-disordered breathing as well as increased sleepiness and BP. Contrary to our hypothesis, there was no significant effect of short-term CPAP withdrawal on daytime CVR as a measure of cerebral endothelial function or on daytime CBF assessed by functional MRI.

A causal relationship between OSA, peripheral endothelial dysfunction and BP has been demonstrated; these measures of cardiovascular risk improve with CPAP treatment [3, 4]. Further evidence from a randomised controlled trial indicated a protective effect of CPAP against severe nocturnal cerebral hypoxia that was similar in magnitude and duration to values causing cerebral dysfunction during unilateral carotid artery clamping during neurosurgery, assessed via near-infrared spectroscopy [20]. Observational studies have also described that the use of CPAP, especially among patients with high treatment adherence, is associated with lower incidence and relative risk reduction of stroke [23, 24]. However, a recent randomised controlled trial did not show a reduction in major cardiovascular endpoints in OSA patients 


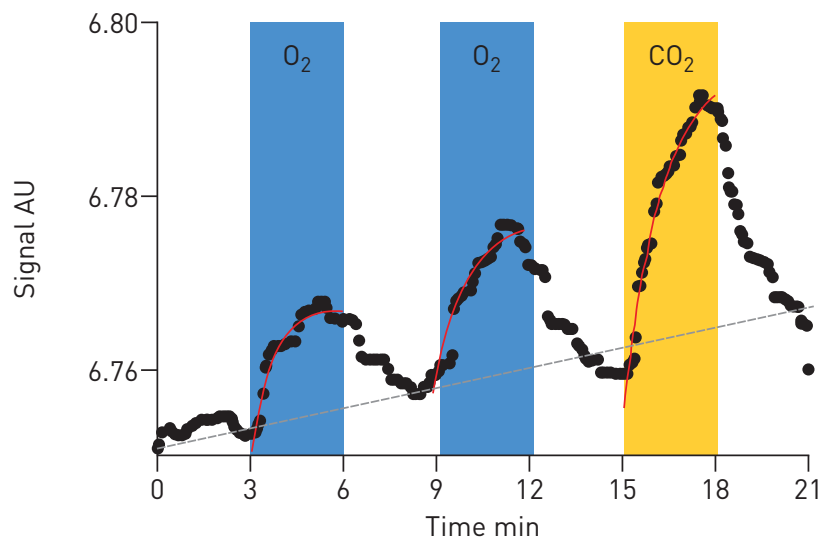

FIGURE 3 Example of a blood oxygen level-dependent (BOLD) signal curve of a random participant. The grey line indicates shift correc-tion of the scanner. The hyperoxic stimuli $\left(\mathrm{O}_{2}\right)$ are presented in blue and the hypercapnic stimulus $\left(\mathrm{CO}_{2}\right)$ in yellow. The red lines are the mathematically fitted curves. The black dots represent the BOLD signal acquisition. The signal change is shown on the $y$-axis in arbitrary units (AU).

with manifest cardiovascular disease allocated to CPAP [25]. Therefore, the beneficial effect of CPAP treatment on major cardiovascular outcomes has been subject to extensive discussion [26, 27].

Several techniques have been used to assess CVR, such as transcranial Doppler ultrasonography (TCD) and positron emission tomography (PET) or single photon emission computed tomography [28-30]. However, MRI allows the entire brain to be mapped with high spatial resolution without the use of radiation, and it has higher reliability compared to ultrasound methods [31,32]. The use of BOLD and ASL MRI enables the investigation of two separate but complementary aspects of vascular regulation. Baseline $\mathrm{CBF}$ is age-dependent and higher in the GM than the WM owing to differences in metabolic demands, neural activity and vascular anatomy. Although hyperoxia is believed to be a mild vasoconstrictor, the absence of negative BOLD signal changes implies that $\mathrm{O}_{2}$-induced vasoconstriction plays a minor role in brain perfusion in contrast to $\mathrm{CO}_{2}$, which is a strong vasodilator [33-35]. In the literature, the average BOLD response to hyperoxia induced by breathing of $100 \% \mathrm{O}_{2}$ in healthy controls is $\sim 1 \%$ in the WM and $\sim 3 \%$ in the GM. The overall signal change induced by hypercapnia in the brain, on the other hand, has a mean magnitude of $2-3 \%[33,36,37]$. Our results are in line with the literature concerning the dependence of the response on the stimulus and tissue type (table 2, figures 2 and 3 ).

Assuming that autonomic impairments could contribute to cerebral injury, MACEY and colleagues [11, 38] performed different tasks (handgrip, cold stimulus, Valsalva manoeuvre) in treatment-naïve OSA patients based on previously reported time-lagged and weaker HR responses to BP changes. Interestingly, they did not find a significant BOLD signal change during the Valsalva manoeuvre in the OSA group compared to healthy controls. Although HR changes occurred during the challenge in the latter study, the BOLD signal response was not delayed, suggesting that cerebral autoregulatory mechanisms may adopt faster than peripheral autonomic regulatory pathways [11]. Thus, our finding of BOLD responses with a mean

TABLE 3 Secondary outcomes

Treatment effect after 2 weeks

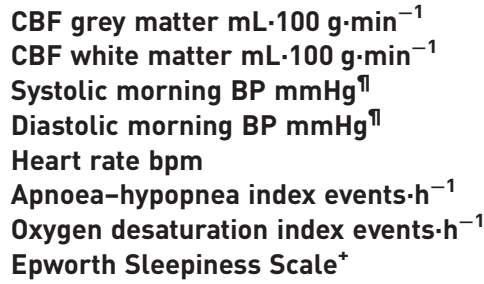
CPAP withdrawal

Adjusted p-value

$\begin{array}{rr}4.20(-0.96-9.36) & 0.110 \\ -0.62(-3.90-2.66) & 0.700 \\ 9.37(1.36-17.39) & 0.023 \\ 7.61(1.40-13.83) & 0.018 \\ 4.01(-0.37-8.40) & 0.071 \\ 38.0(24.2-52.0) & <0.001 \\ 38.0(23.1-53.0) & <0.001 \\ 3.29(0.87-5.72) & 0.009\end{array}$

Data are presented as mean (95\% CI). CPAP: continuous positive airway pressure; CBF: cerebral blood flow; BP: blood pressure; bpm: beats per minute. " : adjusted for baseline; " : BP data from 14 days' home measurements; ${ }^{+}$: Epworth Sleepiness Scale has a maximum of 24 points. 
magnitude comparable to responses described in healthy controls, as a surrogate measure of CVR, could reflect an underlying regulatory mechanism that includes the cerebral vasculature to ensure blood supply and CVR, hitherto not studied in OSA patients.

In contrast to these results, others have described reductions in CVR in the brainstem during swallowing in OSA patients [8]. This finding was interpreted as a potential contributor to the pathogenesis of OSA because altered brainstem CVR may be involved in the control of upper airway muscles. PRILIPKO et al. [13] described significantly higher CVR in several brain regions in healthy subjects compared to age-matched OSA patients. They found that CPAP treatment in the OSA group led to an improvement in CVR but was not associated with a change of CBF after 2 months. However, the observed changes were not homogeneous and did not follow major vascular territories.

GM CBF values reported in two prior studies were comparable with our results $[10,16]$. In addition, decreased as well as increased CBF values in various brain areas of awake, untreated OSA patients assessed by ASL MRI have been described $[9,10,12-14,16]$. We chose to perform ASL measurements assessing $\mathrm{CBF}$ in the absence of evoked responses or challenges. Measuring CBF in this setup is comparable with previous studies $[9,10,12,13,16]$. To further refine detection of brain blood flow changes, taking measurements using ASL during respiratory challenges could provide additional information [17].

When interpreting the BOLD signal response, several important considerations need to be taken into account. $\mathrm{CO}_{2}$ is an important modulator of vascular tone and influences systemic BP via the activation of the sympathetic nervous system, which may in turn affect CVR [39, 40]. To address this issue in TCD studies, the cerebrovascular conductance index has been introduced. This index takes the BP into account by dividing the cerebral artery velocity through the mean arterial BP (MAP) [41]. RYAN et al. [40] described normal hypercapnic cerebrovascular conductance in OSA patients and, in particular, no overnight decline in conductance CVR. Moreover, they did not find any difference between the OSA and healthy control group regarding the MAP response [40]. Others have stated that interpretation of the CVR results after correcting for $\mathrm{BP}$ changes is more accurate in TCD measurements; therefore, continuous monitoring of $\mathrm{BP}$ during the scan is desirable [42]. However, it is important to mention that TCD studies themselves are limited in that they measure blood flow velocity, rather than volumetric flow, which is only representative of blood flow if the diameter of the insonated vessel remains constant $[43,44]$. To date, all studies assessing OSA and CVR via BOLD MRI lack continuous BP recording during the scan $[8,11,13,15,17]$.

Furthermore, the relaxation of vascular smooth vessels and increase in CBF is not always characterised by a linear relationship, but rather a sigmoidal one, with attenuated responses at the extremes, presuming that BP is constant [18]. When vessels are maximally dilated in response to low systemic BP (e.g. hypovolaemia), the vascular response to hypercapnia is subdued. Once the vasodilation mediated through $\mathrm{CO}_{2}$ has reached its limit, increases in perfusion pressure could lead to passive CBF increases [18, 41].

TCD measurements in OSA subjects using the Duffin rebreathing method showed that the maximum end-tidal $\mathrm{CO}_{2}$ tension $\left(\mathrm{PETCO}_{2}\right)$ achieved did not differ between OSA and healthy controls [40]. Although rises in $\mathrm{PETCO}_{2}$ up to $57 \mathrm{mmHg}$ were achieved, most of the responses were not suitable for a sigmoidal fitting [40].

Preliminary results on BOLD signal modelling and vascular resistance describe the possibility of differentiating multiple CBF response patterns based on $\mathrm{CO}_{2}$ stimuli [45]. While this is an interesting research approach, these models assume that MAP, neural activation and metabolism are constant, which is not the case in a clinical setting [46].

There are several further factors potentially contributing to the complexity of this topic, e.g. viscosity and composition of the blood, the role of cardiac output and the rate of cerebral local oxygen consumption [47]. Indeed, direct assessment of CBF, arterial BP and other parameters using invasive methods such as thermal diffusion are only employed in critical ill patients.

We did not measure and adjust for gas concentrations delivered to, or expired by, the subjects. PonsAING et al. [17] measured end-tidal gas concentration during breathing of $5 \% \mathrm{CO}_{2}$-enriched air and breath-holding BOLD MRI. They found no difference between OSA patients and controls in the BOLD response to hypercapnia. Even when adjusting for the change in $\mathrm{O}_{2}$ and $\mathrm{CO}_{2}$ levels of the hypercapnia BOLD CVR response, the result was still not significant. However, for any future studies on this topic, we suggest that concentrations of gases delivered to and expired by the subject should be monitored using computerised gas control systems, which provide precise and repeatable sequences of $\mathrm{PETCO}_{2}$ and $\mathrm{PETO}_{2}$ [17]. Given that MRI measurements were performed in the morning, we cannot exclude the possibility of CBF or CVR impairment during nocturnal apnoeic episodes. Another possible limitation is the withdrawal period of 2 weeks, which might not be sufficient to show the full extent of OSA recurrence and its consequences on CVR and CBF. Furthermore, the current trial population only consisted of a selected 
group of patients with vascular risk factors (i.e. hypertension, dyslipidaemia, diabetes) and optimal therapy compliance, but without any known major cerebral vascular pathologies or history of stroke. Thus, there might be a different response to CPAP withdrawal in patients with previous cerebrovascular events.

In conclusion, despite the recurrence of OSA and its immediate effects on BP, we found no effect of CPAP withdrawal on CVR or CBF assessed by functional MRI.

In particular, daytime CVR did not show any significant reduction after 2 weeks of CPAP withdrawal, assuming that CVR regulation outlasts other pathophysiological effects of OSA in the short term. We suggest that other mechanisms besides changes in CVR must be considered to contribute to the increased risk of stroke.

Acknowledgements: The authors would like to thank Petros Martirosian, Tsogyal Latshang, Sarosh Irani, Lunge Zurich, Lungenliga Glarus and the technicians of the sleep laboratory and the department of radiology for their help in running the programme. Furthermore, we would like to thank Thomas Gaisl and our research group for technical support and constructive criticism. We also thank the patients who participated and often travelled long distances.

Author contributions: Conception and design: M. Kohler, J.R. Stradling, E.I. Schwarz, A. Boss. Funding: M. Kohler. Trial conduct: S. Thiel, F. Lettau, P. Rejmer, A.S. Stöberl, A.S. Becker, N.A. Sievi. Analysis and interpretation of data: S. Thiel, C. Rossi, S. Winklhofer, S.R. Haile, M. Kohler. Drafting the article: S. Thiel. Revising the article for important intellectual content and final approval: all authors.

Conflict of interest: S. Thiel has nothing to disclose. F. Lettau has nothing to disclose. P. Rejmer has nothing to disclose. C. Rossi has nothing to disclose. S.R. Haile has nothing to disclose. E.I. Schwarz has nothing to disclose. A.S. Stöberl has nothing to disclose. N.A. Sievi has nothing to disclose. A. Boss has nothing to disclose. A.S. Becker has nothing to disclose. S. Winklhofer has nothing to disclose. J.R. Stradling reports personal fees for consultancy from Resmed UK and Bayer Germany, outside the submitted work. M. Kohler reports grants from University of Zurich, Lunge Zurich and Swiss National Science Foundation, during the conduct of the study; and grants from Bayer AG, outside the submitted work.

Support statement: This study was supported by grants from the Swiss National Science Foundation (grant no. 32003B_143365/1), Lunge Zurich and the University of Zurich Clinical Research Priority Program Sleep and Health. The funding sources had no role in the design and conduct of the study; in the collection, analysis and interpretation of the data; nor in the preparation, review or approval of the manuscript. Funding information for this article has been deposited with the Crossref Funder Registry.

\section{References}

1 Heinzer R, Vat S, Marques-Vidal P, et al. Prevalence of sleep-disordered breathing in the general population: the HypnoLaus study. Lancet Respir Med 2015; 3: 310-318.

2 Peppard PE, Young T, Barnet JH, et al. Increased prevalence of sleep-disordered breathing in adults. Am J Epidemiol 2013; 177: 1006-1014.

3 Kohler M, Craig S, Pepperell JCT, et al. CPAP improves endothelial function in patients with minimally symptomatic OSA: results from a subset study of the MOSAIC trial. Chest 2013; 144: 896-902.

4 Schwarz EI, Schlatzer C, Rossi VA, et al. Effect of CPAP withdrawal on BP in OSA: data from three randomized controlled trials. Chest 2016; 150: 1202-1210.

5 Khazaie H, Veronese M, Noori K, et al. Functional reorganization in obstructive sleep apnoea and insomnia: a systematic review of the resting-state fMRI. Neurosci Biobehav Rev 2017; 77: 219-231.

6 Loke YK, Brown JW, Kwok CS, et al. Association of obstructive sleep apnea with risk of serious cardiovascular events: a systematic review and meta-analysis. Circ Cardiovasc Qual Outcomes 2012; 5: 720-728.

7 Arzt M, Young T, Finn L, et al. Association of sleep-disordered breathing and the occurrence of stroke. Am J Respir Crit Care Med 2005; 172: 1447-1451.

8 Buterbaugh J, Wynstra C, Provencio N, et al. Cerebrovascular reactivity in young subjects with sleep apnea. Sleep 2015; 38: 241-250.

9 Chen HL, Lin HC, Lu CH, et al. Systemic inflammation and alterations to cerebral blood flow in obstructive sleep apnea. J Sleep Res 2017; 26: 789-798.

10 Innes CR, Kelly PT, Hlavac M, et al. Decreased regional cerebral perfusion in moderate-severe obstructive sleep apnoea during wakefulness. Sleep 2015; 38: 699-706.

11 Macey PM, Kumar R, Ogren JA, et al. Global brain blood-oxygen level responses to autonomic challenges in obstructive sleep apnea. PLoS One 2014; 9: e105261.

12 Nie S, Peng DC, Gong HH, et al. Resting cerebral blood flow alteration in severe obstructive sleep apnoea: an arterial spin labelling perfusion fMRI study. Sleep Breath 2017; 21: 487-495.

13 Prilipko O, Huynh N, Thomason ME, et al. An fMRI study of cerebrovascular reactivity and perfusion in obstructive sleep apnea patients before and after CPAP treatment. Sleep Med 2014; 15: 892-898

14 Yadav SK, Kumar R, Macey PM, et al. Regional cerebral blood flow alterations in obstructive sleep apnea. Neurosci Lett 2013; 555: 159-164.

15 Ryan CM, Battisti-Charbonney A, Sobczyk O, et al. Evaluation of cerebrovascular reactivity in subjects with and without obstructive sleep apnea. J Stroke Cerebrovasc Dis 2018; 27: 162-168.

16 Palomares JA, Tummala S, Wang DJ, et al. water exchange across the blood-brain barrier in obstructive sleep apnea: an MRI diffusion-weighted pseudo-continuous arterial spin labeling study. J Neuroimaging 2015; 25: 900-905.

17 Ponsaing LB, Lindberg U, Rostrup E, et al. Impaired cerebrovascular reactivity in obstructive sleep apnea: a case-control study. Sleep Med 2018; 43: 7-13. 
18 Moreton FC, Dani KA, Goutcher C, et al. Respiratory challenge MRI: practical aspects. Neuroimage Clin 2016; 11: 667-677.

19 Spano VR, Mandell DM, Poublanc J, et al. $\mathrm{CO}_{2}$ blood oxygen level-dependent MR mapping of cerebrovascular reserve in a clinical population: safety, tolerability, and technical feasibility. Radiology 2013; 266: 592-598.

20 Schwarz EI, Furian M, Schlatzer C, et al. Nocturnal cerebral hypoxia in obstructive sleep apnoea: a randomised controlled trial. Eur Respir J 2018; 51: 1800032.

21 Jennum P, Borgesen SE. Intracranial pressure and obstructive sleep apnea. Chest 1989; 95: $279-283$.

22 Berry RB, Budhiraja R, Gottlieb DJ, et al. Rules for scoring respiratory events in sleep: update of the 2007 AASM Manual for the Scoring of Sleep and Associated Events. Deliberations of the Sleep Apnea Definitions Task Force of the American Academy of Sleep Medicine. J Clin Sleep Med 2012; 8: 597-619.

23 Marin JM, Carrizo SJ, Vicente E, et al. Long-term cardiovascular outcomes in men with obstructive sleep apnoea-hypopnoea with or without treatment with continuous positive airway pressure: an observational study. Lancet 2005; 365: 1046-1053.

24 Khan SU, Duran CA, Rahman H, et al. A meta-analysis of continuous positive airway pressure therapy in prevention of cardiovascular events in patients with obstructive sleep apnoea. Eur Heart J 2018; 39: 2291-2297.

25 McEvoy RD, Antic NA, Heeley E, et al. CPAP for prevention of cardiovascular events in obstructive sleep apnea. N Engl J Med 2016; 375: 919-931.

26 McEvoy RD, Kohler M. Con: continuous positive airway pressure and cardiovascular prevention. Eur Respir J 2018; 51: 1702721

27 Martinez-Garcia MA, Campos-Rodriguez F, Javaheri S, et al. Pro: continuous positive airway pressure and cardiovascular prevention. Eur Respir J 2018; 51: 1702400.

28 Minhas PS, Smielewski P, Kirkpatrick PJ, et al. Pressure autoregulation and positron emission tomography-derived cerebral blood flow acetazolamide reactivity in patients with carotid artery stenosis. Neurosurgery 2004; 55: 63-67.

29 Ogasawara K, Yukawa H, Kobayashi M, et al. Prediction and monitoring of cerebral hyperperfusion after carotid endarterectomy by using single-photon emission computerized tomography scanning. J Neurosurg 2003; 99: 504-510.

30 Morgan BJ, Reichmuth KJ, Peppard PE, et al. Effects of sleep-disordered breathing on cerebrovascular regulation: a population-based study. Am J Respir Crit Care Med 2010; 182: 1445-1452.

31 Krainik A, Villien M, Tropres I, et al. Functional imaging of cerebral perfusion. Diagn Interv Imaging 2013; 94: 1259-1278

32 Pillai JJ, Mikulis DJ. Cerebrovascular reactivity mapping: an evolving standard for clinical functional imaging. AJNR Am J Neuroradiol 2015; 36: 7-13

33 Losert C, Peller M, Schneider P, et al. Oxygen-enhanced MRI of the brain. Magn Reson Med 2002; 48: 271-277.

34 Bright MG, Bulte DP, Jezzard P, et al. Characterization of regional heterogeneity in cerebrovascular reactivity dynamics using novel hypocapnia task and BOLD fMRI. NeuroImage 2009; 48: 166-175.

35 Fierstra J, Sobczyk O, Battisti-Charbonney A, et al. Measuring cerebrovascular reactivity: what stimulus to use? J Physiol 2013; 591: 5809-5821.

36 Goode SD, Krishan S, Alexakis C, et al. Precision of cerebrovascular reactivity assessment with use of different quantification methods for hypercapnia functional MR imaging. AJNR Am J Neuroradiol 2009; 30: $972-977$.

37 Kastrup A, Kruger G, Neumann-Haefelin T, et al. Assessment of cerebrovascular reactivity with functional magnetic resonance imaging: comparison of $\mathrm{CO}_{2}$ and breath holding. Magn Reson Imaging 2001; 19: 13-20.

38 Macey PM, Kumar R, Woo MA, et al. Heart rate responses to autonomic challenges in obstructive sleep apnea. PloS One 2013; 8: e76631.

39 Kety SS, Schmidt CF. The effects of altered arterial tensions of carbon dioxide and oxygen on cerebral blood flow and cerebral oxygen consumption of normal young men. J Clin Invest 1948; 27: 484-492.

40 Ryan CM, Battisti-Charbonney A, Sobczyk O, et al. Normal hypercapnic cerebrovascular conductance in obstructive sleep apnea. Respir Physiol Neurobiol 2014; 190: 47-53.

41 Regan RE, Fisher JA, Duffin J. Factors affecting the determination of cerebrovascular reactivity. Brain Behav 2014 4: 775-788.

42 Prakash K, Chandran DS, Khadgawat R, et al. Correction for blood pressure improves correlation between cerebrovascular reactivity assessed by breath holding and $6 \% \mathrm{CO}_{2}$ breathing. J Stroke Cerebrovasc Dis 2014; 23: 630-635.

43 Verbree J, Bronzwaer AS, Ghariq E, et al. Assessment of middle cerebral artery diameter during hypocapnia and hypercapnia in humans using ultra-high-field MRI. J Appl Physiol 2014; 117: 1084-1089.

44 Coverdale NS, Gati JS, Opalevych O, et al. Cerebral blood flow velocity underestimates cerebral blood flow during modest hypercapnia and hypocapnia. J Appl Physiol 2014; 117: 1090-1096.

45 Duffin J, Sobczyk O, Crawley A, et al. The role of vascular resistance in BOLD responses to progressive hypercapnia. Hum Brain Mapp 2017; 38: 5590-5602.

46 Duffin J, Sobczyk O, McKetton L, et al. Cerebrovascular resistance: the basis of cerebrovascular reactivity. Front Neurosci 2018; 12: 409.

47 Gauthier CJ, Fan AP. BOLD signal physiology: models and applications. NeuroImage 2018; in press [https://doi. org/10.1016/j.neuroimage.2018.03.018]. 